\title{
Central Nervous System Delivery and Biodistribution Analysis of an Antibody-Enzyme Fusion for the Treatment of Lafora Disease
}

\author{
Grant L. Austin ${ }^{1}$, Zoe R. Simmons ${ }^{1}$, Jack E. Klier ${ }^{1}$, Alberto Rondon ${ }^{1}$, Brad L. Hodges ${ }^{2}$, \\ Robert Shaffer ${ }^{2}$, Nadine M. Aziz ${ }^{2}$, Tracy R. McKnight ${ }^{2}$, James R. Pauly ${ }^{3}$, Dustin Armstrong ${ }^{2}$, \\ Craig W. Vander Kooi ${ }^{1}$, Matthew S. Gentry ${ }^{1,4,5},{ }^{*}$ \\ ${ }^{1}$ Department of Molecular and Cellular Biochemistry, University of Kentucky College of Medicine, \\ Lexington, KY 40536, USA \\ 2Valerion Therapeutics, Concord, MA 01742, USA \\ ${ }^{3}$ Department of Pharmaceutical Sciences, University of Kentucky College of Pharmacy, \\ Lexington, KY 40536, USA \\ ${ }^{4}$ Lafora Epilepsy Cure Initiative, University of Kentucky College of Medicine, Lexington, KY 40536, \\ USA
}

${ }^{5}$ University of Kentucky Epilepsy Research Center, University of Kentucky College of Medicine, Lexington, KY 40536, USA

\begin{abstract}
Lafora disease (LD) is a fatal juvenile epilepsy, characterized by the accumulation of aberrant glucan aggregates called Lafora Bodies (LBs). Delivery of protein-based therapeutics to the central nervous system (CNS) for the clearance of LBs remains a unique challenge in the field. Recently, a humanized antigen-binding fragment $(\mathrm{hFab})$ derived from a murine systemic lupus erythematosus DNA autoantibody (3E10) has been shown to mediate cell penetration and has been proposed as a broadly applicable carrier to mediate cellular targeting and uptake. We report studies on the efficacy and CNS delivery of VAL-0417, an antibody-enzyme fusion composed of the 3E10 $\mathrm{hFab}$ and human pancreatic a-amylase, in a mouse model of LD. An enzyme-linked immunosorbent assay (ELISA) has been developed to detect VAL-0417 post treatment as a measure of delivery efficacy. We demonstrate the robust and sensitive detection of the fusion protein in multiple tissue types. Using this method, we measured biodistribution in different methods of delivery. We found intracerebroventricular administration (ICV) provided robust CNS delivery when compared to intrathecal administration (IT). These data define critical steps in the translational pipeline of VAL-0417 for the treatment of LD.
\end{abstract}

\section{Graphical Abstract}

* correspondence should be addressed: matthew.gentry@uky.edu, Department of Molecular and Cellular Biochemistry, University of Kentucky College of Medicine, Lexington, KY 40536, USA. phone: 859.323.8482.

The manuscript was written through contributions of all authors. All authors have given approval to the final version of the manuscript. 


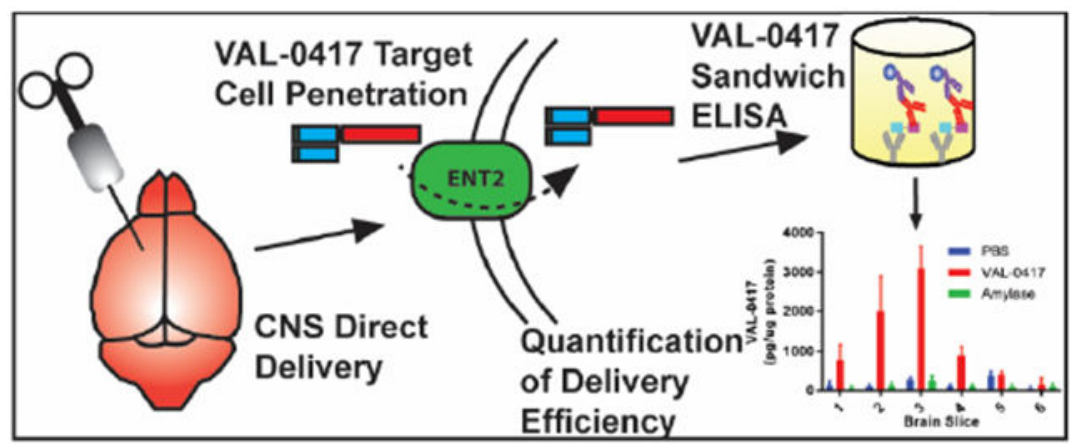

\section{Keywords}

Lafora Disease; Lafora Bodies; Antibody therapy; Amylase; 3E10; CNS Delivery; Autoantibody; ELISA; Glycogen Storage Disease; VAL-0417

\section{Introduction:}

Lafora disease (LD, OMIM \#254789) is an autosomal recessive fatal epilepsy that is also classified as a glycogen storage disease (GSD) ${ }^{1-3}$ There is currently no therapy, treatment, or cure for LD. Patients present with myoclonic, tonic-clonic, and focal occipital seizures caused by a build-up of abnormal, cytoplasmic glycogen-like aggregates called Lafora Bodies (LBs). ${ }^{2-4}$ LBs form in cells from nearly all tissues but are most insidious in neurons and astrocytes where they cause neurodegeneration. ${ }^{5,6} \mathrm{LD}$ is the result of mutations in either Epilepsy, Progressive Myoclonus type 2A (EPM2A) or EPM2B, encoding the glycogen phosphatase laforin and the E3-ubiquitin ligase malin, respectively. ${ }^{1,7-9}$

LBs are comprised of glucose connected by a-1,4- and a-1,6-linkages and synthesized by the same enzymes as glycogen; however, the glucose chains are longer, less branched, and possess higher levels of covalent phosphate. ${ }^{10,11}$ These characteristics cause the molecules to become starchlike and aggregate into pathogenic LBs. Multiple laboratories using diverse systems have established a causal link between LB formation and LD pathology, leading to the current consensus that LBs directly drive neurodegeneration in both LD and non-LD models. ${ }^{12-17}$ Therefore, an immediate goal for LD treatment is to clear LBs and/or prevent their accumulation. Currently, multiple paths to inhibit LB formation and/or clear LBs once they are formed are being pursued. ${ }^{18,19}$ One therapeutic method utilizes antibody-enzyme fusions (AEFs), proteins comprised of an antibody domain fused to an enzyme by a linker peptide, that integrate the required functionalities to degrade LBs and penetrate cells. ${ }^{20,21}$

Cytosolic targeting for protein therapeutics is challenging, but recent antibody-based delivery platforms provide a unique opportunity to penetrate the cell membrane. ${ }^{22-29}$ The monoclonal anti-DNA autoantibody 3E10, produced in a murine model of systemic lupus erythematosus, has been shown to penetrate cells. ${ }^{30}$ Importantly, 3E10 penetrates primary neurons in culture. ${ }^{31}$ Additionally, its antigen-binding fragment (Fab) and its variable domain (Fv) fragment can be fused to an enzyme and promote cytosolic delivery in multiple cell types. ${ }^{25-29} 3 \mathrm{E} 10$ fusions penetrate cells using a nearly ubiquitously expressed 
nucleotide salvage pathway mediated by the equilibrative nucleoside transporter 2 (ENT2, SLC29A2). ${ }^{23,24,30,32}$ Proteins with a large molecular weight, and those not able to traverse membranes on their own, enter cells expressing ENT2 when they are linked to 3E10 fusions. 25-27 $3 \mathrm{E} 10$ alone is also an effective co-therapy against certain cancers when used with DNA damaging treatments, i.e. Doxorubicin or radiation ${ }^{29}$, and $3 \mathrm{E} 10$ has been shown to be safe in phase 1 clinical trials. ${ }^{33}$

The AEF VAL-0417 was generated using the $3 \mathrm{E} 10 \mathrm{hFab}$, capable of neuron penetration and well-suited for clinical use, genetically fused with human pancreatic a-amylase, an enzyme that degrades LBs ${ }^{34}$ Recent work demonstrated that VAL-0417 degrades LBs in vitro and in vivo. ${ }^{35}$ As a key tool to allow monitoring and iterative design of a dosage protocol, we developed an indirect sandwich enzyme-linked immunosorbent assay (ELISA) to specifically detect and monitor VAL-0417. The assay utilizes a capture antibody specific for the DNA binding region of VAL-0417 (anti-3E10 Fab) and allows for orthogonal detection of the fused amylase. The ELISA yields accurate quantification of VAL-0417 delivered to tissues or circulating in the blood, even in tissues with high endogenous amylase activity. Furthermore, straightforward modifications to this ELISA will allow the development of ELISAs for other biologicals using the 3E10-based AEF delivery platform. Using this assay, we define the appropriate method of VAL-0417 central nervous system (CNS) delivery. Administration of VAL-0417 into the CNS via the spinal cord or subarachnoid space, i.e. intrathecal administration, resulted in only moderate detection in the brain and mostly in the caudal regions. In contrast, we find that administration directly into the brain ventricles by intracerebroventricular administration allows more efficient CNS delivery of VAL-0417.

\section{Experimental Section:}

\section{Generation of VAL-0417:}

VAL-0417 was designed by Valerion Therapeutics (Concord, MA) and produced and purified by Proteos (Kalamazoo, MI) as previously described. ${ }^{35}$ A gene fusion of the codon optimized genes for human IgG2A 3E10 Fab heavy chain followed by a linker and AMY2A, and the 3E10 kappa light chain were synthesized and cloned into a proprietary version of the pTT5 plasmid (Canadian National Research Centre). This plasmid was transfected into HEK293-6E cells, which grew for 5 days before VAL-0417 was purified using pre-packed CaptureSelect IgG-CH1 affinity columns (Thermo Fisher). The purified VAL-0417 was dialyzed and concentrated to $10 \mathrm{mg} / \mathrm{mL}$ before being snap frozen and stored in dialysis buffer (20 mM histidine, $150 \mathrm{mM} \mathrm{NaCl}, \mathrm{pH} 6.5)$ at $-80^{\circ} \mathrm{C}$.

\section{In vitro and ex vivo degradation assays:}

Lafora bodies were purified using established methods. ${ }^{35,36}$ Briefly, Epm2a-/- mouse brains were harvested and homogenized in lysis buffer (100 mM Tris- $\mathrm{HCl} \mathrm{pH}$ 8.0, $200 \mathrm{mM}$ $\mathrm{NaCl}, 1 \mathrm{mM} \mathrm{CaCl} 2,0.5 \% \mathrm{NaN}_{3}$ ) and then centrifuged for $10 \mathrm{~min}$ at $10,000 \mathrm{xg}$ at $4{ }^{\circ} \mathrm{C}$. The pellet was collected and resuspended in lysis buffer with $0.2 \%$ SDS and $0.4 \% \mathrm{mg} / \mathrm{ml}$ proteinase $\mathrm{K}$ and digested overnight at $37^{\circ} \mathrm{C}$. Following digestion, the suspension was filtered through $140 \mu \mathrm{m}$ and $60 \mu \mathrm{m}$ nylon filters and then centrifuged for $5 \mathrm{~min}$ at 16,000 xg. The pellet containing LBs was washed and then resuspended in LB buffer (10 mM HEPES- 
$\mathrm{KOH} \mathrm{pH}$ 8.0, 0.1\% $\mathrm{NaN}_{3}$ ). LB yield was quantified using the Pflüger method ${ }^{37}$ followed by acid hydrolysis and glucose determination using the R-Biopharm Inc glucose assay kit (Roche 10716251035).

$50 \mu \mathrm{g}$ of LBs were incubated with either PBS or $10 \mu \mathrm{g}$ VAL-0417 to a final volume of 100 $\mu \mathrm{L}$ in citrate/phosphate buffers ranging from $\mathrm{pH} 4.5$ to $\mathrm{pH} 7.0$ for $6 \mathrm{~h}$ with shaking at $37^{\circ} \mathrm{C}$. LBs were then pelleted by centrifugation for $5 \mathrm{~min}$ at $16,000 \mathrm{xg}$. Following centrifugation, $75 \mu \mathrm{L}$ of the supernatant was removed and a glucose determination assay was performed using the Boehringer-Manheim D-glucose kit (R-BioPharm). The $25 \mu \mathrm{L}$ remaining was mixed with 20x Lugol's solution and the stained LBs were visualized using Nikon Eclipse E600 using DIC/Nomarski contrast and an AxioCam MRm camera.

Degradation of native LBs in tissue was performed using $6 \mu \mathrm{m}$ formalin fixed and paraffin embedded Epm2a--/- mouse brain slices mounted on glass slides. The slides were deparaffinized and then incubated in BioVision amylase activity assay buffer solution containing PBS, VAL-0417, or diastase (Sigma 09962-100G) for $3 \mathrm{~h}$. Both VAL-0417 and diastase were used at $5 \mathrm{mg} / \mathrm{mL}$. The digestion was terminated by rinsing the slides with water. The slides were then stained with periodic acid-Schiff (PAS) and visualized and quantified using a slide scanner and HALO image analysis software (PerkinElmer). Quantification was done by taking three regions rich in LBs in the untreated slice (thalamus, inferior colliculus, and brain stem) and dividing these regions into six equally sized fields. The LBs in each field were counted and the average number of LBs were normalized to the untreated slice for each region.

\section{SDS-PAGE and Western blot:}

SDS-PAGE and Western blots were run using TGX stain-free pre-cast 10\% gels (Bio-Rad 456-8034). Western transfers were performed using a semi-dry turbo transfer system onto PVDF membranes (Bio-Rad 170-4274). The membranes were blocked with 5\% non-fat milk and probed with primary antibodies [rabbit anti-pancreatic amylase (Abcam ab21156, lot GR283033-25) and anti-3E10 Fab (ab10A4, anti-id, Valerion Therapeutics)] at 1/5000 and 1/500 dilutions, respectively. The anti-3E10 Fab antibody is a mouse monoclonal antibody against the DNA binding region of the 3E10 antibody, Fab, and Fv. It is similar to the previously described anti-id antibodies against $3 \mathrm{E} 10 .{ }^{38}$ Membranes were incubated with the secondary antibodies [goat anti-rabbit IgG, HRP-linked Antibody (Cell Signaling Technology 7074, lot 27) and goat anti-mouse IgG, HRP-linked antibody (Invitrogen 62-6520, lot SG253194)] at 1/7500 dilution. The membranes were developed using chemiluminescence. Gels and membranes were imaged on a ChemiDoc MP imaging system (Bio Rad 12003154).

\section{Enzyme-Linked Immunosorbent Assay:}

Wells of a Corning 9018 96-well plate were incubated with $100 \mu \mathrm{L}$ of capture antibody (ab10A4) overnight ( $16 \mathrm{~h}$ ) at a final concentration of $2 \mu \mathrm{g} / \mathrm{mL}$ in TBS at $4^{\circ} \mathrm{C}$ in a humidified chamber. Wells were then rinsed 3 times with $200 \mu \mathrm{L}$ TBS followed by incubation for $1 \mathrm{~h}$ with $200 \mu \mathrm{L}$ of blocking solution (5\% non-fat milk in TBS) at room temperature followed by wells being rinsed 3 times with $200 \mu \mathrm{L}$ TBS. $100 \mu \mathrm{L}$ of sample 
(tissue or recombinant protein sample diluted to $100 \mu \mathrm{L}$ in TBS) was added and incubated for $1 \mathrm{~h}$ at room temperature, wells were rinsed 3 times with $200 \mu \mathrm{L}$ of TBS, and $100 \mu \mathrm{L}$ of primary antibody (Abcam ab21156) in 5\% non-fat milk in 1xTBS was added and incubated for $1 \mathrm{~h}$ at room temperature. The wells were washed (solution added and incubated for $5 \mathrm{~min}$ before being removed) 3 times with TBS. $100 \mu \mathrm{L}$ of secondary antibody (Cell Signaling Technology 7074) in 5\% non-fat milk in TBS was added and incubated for $1 \mathrm{~h}$ at room temperature. The wells were washed 5 times with TBS and $100 \mu \mathrm{L}$ of TMB substrate (Thermo Fisher N301, lot TA257953) was added and developed according to the manufacturer's instructions. The reaction was terminated by adding $100 \mu \mathrm{L}$ of stopping solution $\left(0.18 \mathrm{M} \mathrm{H}_{2} \mathrm{SO}_{4}\right)$. Absorbance was quantified at $450 \mathrm{~nm}$ using a BioTek Epoch2 microplate reader.

To quantify the anti-3E10 Fab bound to the plate, goat anti-mouse IgG, HRP-linked antibody (Invitrogen 62-6520) was used at a 1/5000 dilution and developed using TMB substrate (Thermo Fisher).

\section{Amylase Activity Assay:}

Amylase activity assays were performed according to the manufacturer's protocol (BioVision K711-100). Briefly, samples were homogenized in amylase assay buffer (BioVision, K711-100-1). Following homogenization, the lysate was diluted and incubated with a reaction mixture containing ethylidene-pNP-G7 (the substrate; ethylidene capped heptaose linked to p-nitrophenol) and porcine glucosidase for $1 \mathrm{~h}$. During the incubation, absorbance readings were quantified every minute at $405 \mathrm{~nm}$. Enzyme activity was determined using the slope generated from these readings. The homogenized samples were also analyzed in the ELISA and BCA assays.

\section{BCA Assay:}

Protein concentrations were determined using the Pierce BCA Protein Assay Kit (Thermo Scientific 23227) according to the manufacturer's protocol.

\section{Mouse treatment and tissue harvesting:}

All procedures were approved by the Institutional Animal Care and Use Committee (IACUC) as specified by the 1985 revision of the Animal Welfare Act.

Blood was obtained via a sub-mandibular artery bleed from untreated wild type (WT) mice and whole blood was collected in EDTA coated tubes (BD Microtainer 365974). Blood was diluted to the appropriate volume in 0.25M EDTA in PBS to yield a solution that was $80 \%$ whole blood. Mice were euthanized by cervical dislocation, a cranial dissection was performed to collect the brain, and the brain was homogenized in BioVision amylase assay buffer.

WT mice were treated with $0.08 \mathrm{mg} /$ day of VAL-0417 or PBS for 14 days via intracerebroventricular (ICV) and then sacrificed on day 15. An additional cohort of WT mice were treated with $0.08 \mathrm{mg} /$ day of VAL-0417 or PBS for 14 days via intrathecal (IT) administration and then sacrificed on day 15. Briefly, ICV administration involved a 
craniotomy and insertion of a cannula with osmotic pump (ALZET®; DURECT

Corporation) into the target ventricle. The catheter was anchored, and the pump was deposited in a dorsal subcutaneous pocket. IT administration involved surgical exposure of the cisterna magna. A catheter was guided through the lumbar region with a stylet. The catheter was connected to an osmotic pump (ALZET®; DURECT Corporation). The catheter was anchored, and the pump was deposited in a dorsal subcutaneous pocket. After euthanasia, a cranial dissection was performed to collect the brain and the brain was sectioned into 6 sections before being frozen in liquid nitrogen.

LD mice $^{14}$ (Epm2a--/-) were administered VAL-0417, recombinant pancreatic amylase, or PBS via ICV administration. LD mice were treated with $0.08 \mathrm{mg} /$ day of VAL-0417 or 0.04 $\mathrm{mg} /$ day recombinant pancreatic amylase for 28 days and then sacrificed on day 29 . Brains were harvested and quickly sectioned into 6 sections before being frozen in liquid nitrogen. Brains from two of each type of treated LD mice and two WT mice treated for 28 days with PBS were formalin fixed and paraffin embedded before undergoing immunohistochemical analysis using the a-amylase antibody at $5 \mu \mathrm{g} / \mathrm{mL}$ (Abcam ab21156).

Brain surgeries, implantation of catheters, sacrificing of treated animals, brain sectioning, and brain fixation was performed by Northern Biomedical Research (NBR, Norton Shores, MI). Samples from NBR were shipped on dry ice and homogenized in BioVision amylase assay buffer.

Untreated LD mice brains were harvested and then either homogenized in lysis buffer for LB purification or formalin fixed. The fixed brains were paraffin embedded, $6 \mu \mathrm{m}$ slices were generated, and slices were mounted on glass slides for ex vivo enzymatic LB digestion and PAS staining.

\section{Statistics:}

Statistical analyses were performed using GraphPad Prism version 7.03 using parameters from previous studies. ${ }^{39},{ }^{40}$ Error is presented as Standard Error of the Mean (SEM), unless otherwise stated.

\section{Results:}

\section{Assessment of VAL-0417 and Optimization of a VAL-0417 ELISA}

VAL-0417 is an AEF candidate for the treatment of LD. VAL-0417 was generated by fusing the genes for the heavy chain $\mathrm{hFab}$ fragment of antibody 3E10 and pancreatic a-amylase and coexpressing with the light chain (Figure 1A). The suitability of VAL-0417 was first assessed in vitro. LBs were purified from the brain of Epm2a-/- mice, incubated with VAL-0417 for $6 \mathrm{~h}$ in a range of $\mathrm{pH}$ buffers, samples were centrifuged to pellet undigested material, and the amount of glucose released was quantified from the supernatant (Figure S1 and 1B). VAL-0417 degradation increased in a pH dependent manner with maximal degradation at $\mathrm{pH}$ 7.0. In addition to biochemically defined degradation, LBs were stained with Lugol's solution after incubation with VAL-0417 or buffer. The morphology and quantity of the LBs is dramatically changed with VAL-0417 treatment when visualized by light microscopy (Figure 1C). Next, we sought to test VAL-0417 activity ex vivo. Brains 
from 9-month old Epm2a-/-mice were fixed, slices were generated and mounted on slides, and the slides were treated with buffer or VAL-0417. Following treatment, the slides were PAS-stained, and the slides were analyzed using the HALO digital analysis platform for PAS + LBs (Figure 1D). Slices treated with VAL-0417 were nearly devoid of PAS+ LBs, while the untreated and buffer treated samples exhibited high abundance of PAS+ LBs in the thalamus, inferior colliculus, and the brain stem (Figure 1E).

After in vivo administration, VAL-0417 cannot be distinguished from endogenous amylase using anti-amylase antibodies or amylase activity assays. Additionally, a mouse monoclonal anti-3E10 Fab antibody is not robust enough for accurate quantification of low $\mathrm{hFab}$ fusion concentrations that are present in tissue lysates. To address these deficiencies, a sandwich ELISA combining an anti-Fab capture antibody that is specific for 3E10 and a polyclonal rabbit anti-amylase primary detection antibody that provides signal amplification was developed (Figure 2A). This combination of antibodies provides both specificity and robust signal for VAL-0417 detection.

It was first necessary to determine whether the proposed detection and capture antibodies would specifically detect VAL-0417. Recombinant VAL-0417, salivary a-amylase, and pancreatic a-amylase were resolved on a non-reducing SDS-PAGE, a Western transfer was performed, and the blot was probed with anti-pancreatic a-amylase (ab21156) or anti-3E10 Fab (ab10A4). The anti-pancreatic a-amylase antibody detected VAL-0417 and the two recombinant free amylases (Figure 2B). Importantly, the anti-3E10 Fab antibody only detected VAL-0417 (Figure 2B). The anti-pancreatic a-amylase antibody detected two bands in the VAL-0417 lane, while the anti-3E10 Fab antibody only detected one band. This difference is because the anti-3E10 Fab antibody only detects intact Fab that contains both the heavy and light chains whereas the anti-pancreatic a-amylase antibody detects VAL-0417 with and without the Fab light chain. The heavy chain of the Fab fused to the amylase is not produced in equal amounts to the light chain of the Fab. Therefore, both the complete VAL-0417 and the independent heavy chain-amylase fusion are purified from the cell culture. When VAL-0417 is resolved on a reducing gel, bands are observed at the expected $75 \mathrm{kDa}$ that corresponds to the fully reduced VAL-0417 and $25 \mathrm{kDa}$ representing the Fab light chain (Figure S2A). When the amylase panel in Figure 2B is run on a reducing gel and transferred to a western blot, probing with the anti-pancreatic $a$-amylase antibody again reveals only a single band at $75 \mathrm{kDa}$ for VAL-0417 (Figure S2B). These data establish the utility of anti-3E10 Fab as a capture antibody and anti-amylase for detection.

The concentrations of capture (anti-3E10 Fab) and detection (anti-pancreatic a-amylase) antibodies were then optimized for use in the proposed ELISA. Increasing amounts of anti-3E10 Fab were bound to wells of a high-binding 96 -well plate from $0 \mathrm{ng} / \mu \mathrm{L}$ to $3 \mathrm{ng} / \mu \mathrm{L}$ to determine the concentration of maximally bound antibody. The anti-3E10 Fab antibody was maximally bound to the plate at a concentration of $2 \mathrm{ng} / \mu \mathrm{L}$ as determined by colorimetric analysis (Figure 2C). Optimization of the rabbit anti-pancreatic a-amylase and goat anti-rabbit IgG HRP detection antibodies was also performed. Titers of rabbit antipancreatic a-amylase and goat anti-rabbit IgG HRP detection antibodies were analyzed while maintaining constant concentrations of the capture antibody, antigen, and other detection antibody. The anti-pancreatic a-amylase and the goat anti-rabbit IgG HRP titers 
indicated a range of optimal combinations, i.e. any concentration within the linear range of the titer could be optimal (Figure 2D \& 2E). Taking three points from the linear range of each titer gave nine possible antibody combinations that were tested using constant capture concentrations. These combinations were tested on high $(\mathrm{H}, 500 \mathrm{pg} / \mu \mathrm{L})$, low $(\mathrm{L}, 10 \mathrm{pg} / \mu \mathrm{L})$ and zero (0) concentrations of VAL-0417 and evaluated on the consistency of the replicates, the lack of background, the ability to detect the low concentration, and the signal range between the low and high concentrations (Figure S3). If results were equal, then the lowest antibody concentration was considered optimal. Cumulatively, these results demonstrated that the optimal antibody dilutions are 1/7500 for the anti-pancreatic a-amylase primary and 1/12500 for the goat anti-rabbit IgG HRP secondary.

The optimized antibody concentrations were then assayed using a series of VAL-0417 concentrations in triplicate ranging from $0.1 \mathrm{pg} / \mu \mathrm{L}$ to $10,000 \mathrm{pg} / \mu \mathrm{L}$ (Figure $3 \mathrm{~A}$ ). A best fit curve was generated to analyze the results using a four-parameter $\operatorname{logistic}^{39}$ (FPL) model (Equation 1). The FPL model was selected because it provides a better fit (percent recovery) to all of the data than the linear models, even in instances where the linear model lack-of-fit value $\left(\mathrm{R}^{2}\right)$ is equal to or greater than $0.99 .{ }^{39}$

$$
\mathrm{Y}=\text { Top }+\frac{(\text { Bottom-Top })}{1+\left(\frac{\mathrm{X}}{\mathrm{EC}_{50}}\right)^{\text {Slope }}}
$$

In the FPL model, Y is the output value, in this case the absorbance at 450nm. Top is the upper asymptote and bottom is the lower asymptote. $\mathrm{X}$ is the concentration of VAL-0417 in the well, $\mathrm{EC}_{50}$ is the concentration with $50 \%$ maximum response, and slope is the Hill coefficient.

Evaluation of the percent recovery of each point with the concentration calculated by the FPL curve established that the lowest concentration measured within $20 \%$ of the FPL curve and that is significantly higher than background is $10 \mathrm{pg} / \mu \mathrm{L}$ or $10 \mathrm{ppm}$ of VAL-0417 (Figure 3B). The upper limit is $5000 \mathrm{pg} / \mu \mathrm{L}$, i.e. the point where the curve is saturated, and absorbance no longer increases with increasing concentration. This model of standard curve with FPL fit is utilized to determine the concentration of VAL-0417 dose delivered.

\section{Tissue Matrix Effects on the VAL-0417 ELISA}

Rapid and reliable analytical tests on whole blood are necessary for patient treatment and diagnosis, and they are important data in clinical trials; however, whole blood is an historically complex matrix for ELISA assays. ${ }^{41}$ We sought to define the performance of the VAL-0417 ELISA in whole mouse blood to establish its reliability on samples in a complex matrix. Assays were performed to generate a standard curve in buffer versus $80 \%$ whole mouse blood using concentrations falling within the linear region of the curve in figure $3 \mathrm{~A}$ (Figure 4). The slopes of the linear standard curves generated in the presence of blood were consistently decreased 3-fold versus standard curves in buffer (Figure 4A), yet they displayed a robust percent recovery throughout most of the standard curve when compared to the FPL curve (Figure 4B \& 4C). The lower limit of detection increased to between 10 and $50 \mathrm{pg} / \mu \mathrm{L}$. These data suggest that analysis of whole blood with this ELISA is viable 
when the standard curve is performed with appropriate referencing. Additionally, optimization of washing and incubation procedures within the ELISA protocol could help reduce these matrix effects. The tissue matrix effect was less pronounced when brain tissue was utilized as the matrix. When assays were performed with and without $10 \mu \mathrm{g}$ protein from whole brain tissue homogenate or $100 \mu \mathrm{g}$ protein from whole brain tissue homogenate, the ELISA standard curve differences between the slopes were $8.89 \%$ and $7.79 \%$, respectively.

To test the specificity of the ELISA in the presence of tissue lysate, an in vitro screen was designed comparing equal mass concentrations of recombinant human pancreatic a-amylase and VAL-0417 with or without $50 \mu \mathrm{g}$ protein from whole brain tissue homogenate. These parameters were chosen to test if the signal from biological samples is exclusively from VAL-0417 or if some signal is from endogenous amylases. The ELISA robustly detected VAL-0417 in the absence and presence of brain tissue homogenate and this signal was not changed by the presence of exogenously added pancreatic a-amylase. Conversely, there was no detectable signal from the sample with no antigen and no signal from the sample that only included pancreatic a-amylase (Figure $\mathrm{S} 4$ ). Thus, the assay robustly differentiates between VAL-0417, pancreatic a-amylase, and endogenous amylases.

\section{Comparison of ICV and IT VAL-0417 Brain Delivery using the VAL-0417 ELISA}

To define an optimal mode of therapy administration, initial pharmaco-delivery studies were performed on two modes of direct CNS drug delivery. Wild type (WT) mice were continuously infused with VAL-0417 (0.08 mg/day) or PBS for 14 days either via intracerebroventricular (ICV) administration or intrathecal (IT) administration. ICV administration is a method of direct infusion into the brain ventricles and IT administration utilizes the cerebrospinal fluid in the spinal canal to deliver treatment to the brain. ICV is more direct, but it is more invasive and requires brain surgery. One day after completion of VAL-0417 or PBS administration, the mice were euthanized, the brains were collected, and each brain sliced laterally into six sections labeled numerically from rostral to caudal (i.e. slice one was the most rostral portion of the cortex and slice six included the cerebellum, Figure 5 inset). In the ICV administered brains, VAL-0417 was detected in all six slices, although only significantly above background in slices 2,3 , and 4 , with a maximal value of $1600 \mathrm{pg} / \mathrm{\mu g}$ protein (Figure 5). The administration site is within slice 3 and this is the same slice that yields the highest levels of VAL-0417. Conversely, IT administration did not yield high levels of VAL-0417 CNS distribution and the maximal ELISA detection was only 240 $\mathrm{pg} / \mu \mathrm{g}$ protein (Figure 5). VAL-0417 was detected throughout the brain after IT administration, but the levels in all slices were dramatically less than with ICV administration after the same treatment length and no slice produced signal significantly higher than background. These results illustrate the utility of the VAL-0417 ELISA for preclinical analyses and the superior delivery via ICV administration compared to IT administration in mice.

\section{CNS Delivery of VAL-0417 using ICV Administration in a LD Model}

Finally, we sought to test the delivery efficacy of VAL-0417 and specificity of the ELISA in comparison with administration of amylase in vivo in an Epm2a-/- LD mouse model. ${ }^{14} \mathrm{LD}$ 
is a fatal, neurodegenerative epilepsy and multiple groups have demonstrated that LBs in neurons and astrocytes drive disease progression. ${ }^{12-17}$ Therefore, VAL-0417 must be delivered to the central nervous system in order to be efficacious. LD mice were treated via ICV administration with PBS, or equal molar concentrations of VAL-0417 (0.08 mg/day) or recombinant pancreatic amylase $(0.04 \mathrm{mg} / \mathrm{day})$. After 28 days of continuous administration, the mice were euthanized, the brains were collected, and each brain was either fixed for immunohistochemical (IHC) analysis or sliced laterally into six sections labeled numerically from rostral to caudal and frozen.

The fixed brains were analyzed by IHC using an a-amylase antibody. Mice treated with VAL-0417 displayed robust intracellular positive staining in the rostral cortex, choroid plexus, medial cortex, and cerebellum (Figure 6A). Conversely, mice treated with a-amylase displayed minimal extracellular positive staining and no robust intracellular positive staining (Figure 6A). These data demonstrate the necessity of the VAL-0417 cell penetration platform.

The frozen brain sections were also analyzed using the VAL-0417 ELISA. Robust levels of VAL-0417 were detected in slices 1-4 with the highest levels being those immediately adjacent to the administration site, i.e. slices two and three (Figure 6B), which is consistent with the ICV VAL-0417 distribution seen in the WT mice (Figure 5). No significant signal was detected for the PBS treated or amylase treated animals in similar slices. These results indicate that VAL-0417 is disseminated throughout the brain during ICV administration and the ELISA robustly detects the AEF. While the ELISA is specific to VAL-0417, the amylase activity assay detects both VAL-0417 and any amylase. To confirm the ELISA results for VAL-0417 and determine distribution of the recombinant amylase, we performed amylase activity assays on samples from each brain slice. These results show similar trends as the ELISA and indicate that VAL-0417 is active in each slice and that the recombinant amylase without the hFab fused to it does not accumulate in the brain (Figure 6C). Therefore, the amylase assay confirmed the efficacy of the ELISA and the importance of the hFab fragment on VAL-0417 for delivery of amylase to the brain. While the amylase activity assay was important in this study to verify the delivery of recombinant amylase, the ELISA is a more optimal assay for the detection of VAL-0417. The ELISA provides less replicate variability, has a lower limit of detection, is more cost effective, and dramatically decreases background when compared to the amylase activity assay. Cumulatively, these results demonstrate that the VAL-0417 ELISA detects VAL-0417 after treatment, the ELISA differentiates between VAL-0417 and endogenous or exogenous amylases from treated samples, and ICV administration of VAL-0417 achieves robust biodistribution throughout the brain in the LD mouse model.

\section{Discussion:}

The development of quantitative detection assays for new therapeutics is an essential aspect of pre-clinical and clinical research. This study focuses on VAL-0417, a 3E10-based cell penetrating AEF that utilizes pancreatic amylase to degrade LBs and is efficacious in preclinical LD mouse models. ${ }^{35}$ We developed an ELISA for VAL-0417 and provided initial preclinical data demonstrating VAL-0417 delivery into WT and LD mice. 
The sandwich ELISA, utilizing the anti-3E10 Fab and anti-pancreatic amylase antibodies, is a simple and robust method of detecting VAL-0417 concentration in samples from in vivo studies. The antibodies used in this ELISA function in combination to produce a robust signal that is specific for VAL-0417. The mouse monoclonal anti-3E10 Fab is highly specific for the DNA binding region of 3E10 and its fragments, but only produces a weak signal when used alone in an antigen-down ELISA format. The polyclonal rabbit anti-pancreatic amylase yields strong detection of amylases but also detects endogenous amylases. Furthermore, due to the interest in using VAL-0417 and related fusions for systemic delivery, these data demonstrate the effect of tissue matrices on VAL-0417 detection. Tissue matrix effects were found to result in systematic effects which can be accounted for with proper assay controls.

This ELISA is a substantial improvement to other methods that could be used for VAL-0417 quantification such as amylase activity assays, western blots, or mass spectrometric approaches. While amylase activity in brain is low, background signal would be a major issue in tissues of the gastrointestinal tract where endogenous amylase activity is high. This issue could be relevant in LD treatment because LBs are found throughout the body including high levels in the liver. ${ }^{42}$ It is currently unclear what the physiological effects of these systemic LBs are because patients die from their severe neurological symptoms before systemic effects are observed. These effects may become pronounced and could require intervention after patients are treated for their brain LBs via direct CNS therapy delivery. The ELISA is also superior because whole tissue lysate can be tested, whereas the amylase assay utilizes either dramatically diluted whole lysate or centrifugally cleared lysate. If the sample must be dramatically diluted, then the lower limit of detection will be increased. Clearing the lysate is also an issue in LD models because the VAL-0417 and all amylases would bind and pellet with LBs.

Two methods of VAL-0417 delivery to the brain were evaluated in this study. While less invasive, the IT mode of administration did not yield as robust of a biodistribution of VAL-0417 throughout the brain in the mouse models. Conversely, ICV administration produced a robust signal after 14 days of administration. While ICV drug administration is invasive, it is already a relatively common procedure in the clinic. ${ }^{43,44} \mathrm{ICV}$ administration of cerliponase alfa for children with CLN2 (neuronal ceroid lipofuscinosis type 2) disease is well tolerated and is becoming the standard of care. ${ }^{45,46}$ Currently, ICV administration is the optimal option for treating devastating neurological diseases like LD; however, novel methods are being developed to deliver biologics across the blood brain barrier (BBB) via noninvasive methods. One mechanism utilizes a modified $\mathrm{Fc}$ antibody fragment to interact with BBB transferrin receptors to facilitate transport via receptor mediated transcytosis. ${ }^{47}$ These emerging technologies provide exciting possibilities for future treatment of neurological diseases with AEFs such as VAL-0417.

LBs are highly prevalent in the cerebral cortex, substantia nigra, and thalamus, which would correspond to slices 3,4 , and 5 in this study, and they are also present in the cerebellum, which would be slice 6 (Figure 5). ${ }^{42}$ The ICV administration site in this study was within a ventricle in slice 3 and the ICV administration achieved a maximum delivery load in this slice. VAL-0417 diffused via roughly Fickian diffusion through the tissue from the injection 
site throughout the entire brain. This diffusion pattern is consistent with prior descriptions of antibody passive diffusion through the extracellular and perivascular spaces of the brain. ${ }^{48,49}$ This diffusion is likely through the perivascular spaces, which provide a less inhibitory environment to convective flow than the dense extracellular matrix in the interstitial space. ${ }^{50}$ Diffusion of $\operatorname{IgG}$ through the brain can be enhanced with co-injection with hyperosmolar mannitol. ${ }^{49}$ Therefore, improvements to the diffusion of VAL-0417 could be observed after co-injection of mannitol, which would allow for a reduced dosing regimen.

More time dependent studies are necessary to elucidate the optimal delivery regimen of VAL-0417. The present studies included dosing durations of two and four weeks. Moving forward, shorter treatment durations as well as intermittent treatment delivery should be tested. As with all therapeutic interventions, it will be necessary to determine the minimum effective dose and treatment duration for ICV administration before transitioning this therapy to the clinic. Additionally, while the IT delivery was not optimal in this study, if the dose needed for therapeutic benefit is found to be lower, then IT administration may be the optimal choice due to the less invasive nature. While IT administration was not optimal in the mouse model, further testing in larger vertebrate models, including rats and dogs, could be explored. Additionally, both administration methods should also be evaluated in nonhuman primates to determine the optimal delivery method for the clinic. Furthermore, methods for penetrating the BBB including Molecular Trojan Horses, intranasal injection, and hyperosmolar BBB disruption are being explored for CNS delivery. ${ }^{51}$ These approaches could be evaluated for VAL-0417. The ELISA method established here will be essential for all future studies evaluating the delivery and pharmacokinetics of VAL-0417.

The results presented herein are encouraging for the use of VAL-0417 as a LD therapy, which currently has no therapy or treatment. These data begin to establish the necessary delivery for VAL-0417 and will guide clinical trials designed to establish the efficacy of VAL-0417 to breakdown LBs in tissue and alleviate the devastating symptoms of LD.

\section{Supplementary Material}

Refer to Web version on PubMed Central for supplementary material.

\section{Acknowledgements:}

The authors would like to thank Dr. Ramon Sun regarding initial conversations for the conception and development of the VAL-0417 ELISA. We would like to thank Jill Zeller and the rest of the NBR team for performing the surgical implantation of the pumps and catheters for the ICV and IT treatments and for harvesting tissue following the completion of the treatments. We also thank Lyndsay Y oung, Kit Donohue, Bobby Murphy, and the rest of the Gentry lab for helpful discussions and assistance during manuscript preparation. Data and materials availability: Researchers may obtain VAL-0417 with a material transfer agreement from Valerion Therapeutics. All reasonable requests for collaboration involving materials used in the research will be fulfilled provided that a written agreement is executed in advance between Valerion Therapeutics and the requester (and his or her affiliated institution). Such inquiries or requests should be directed to D.A.

Funding Sources:

This work was supported by a sponsored project from Valerion Therapeutics, NIH grants R01 NS070899, P01 NS097197, and an Epilepsy Foundation New Therapy Commercialization Grant (all to M.S.G.) and by the Biospecimen Procurement and Translational Pathology Shared Resource Facility of the University of Kentucky Markey Cancer Center (P30CA177558). 


\section{Abbreviations:}

$\begin{array}{ll}\text { LD } & \text { Lafora Disease } \\ \text { LB } & \text { Lafora Body } \\ \text { CNS } & \text { Central Nervous System } \\ \text { GSD } & \text { Glycogen Storage Disease } \\ \text { ELISA } & \text { Enzyme-Linked Immunosorbent Assay } \\ \text { ICV } & \text { Intracerebroventricular } \\ \text { IT } & \text { Intrathecal }\end{array}$

\section{References:}

(1). Gentry MS; Dixon JE; Worby CA Lafora Disease: Insights into Neurodegeneration from Plant Metabolism. Trends Biochem. Sci 2009, 34 (12), 628-639.10.1016/j.tibs.2009.08.002. [PubMed: 19818631]

(2). Gentry MS; Guinovart JJ; Minassian BA; Roach PJ; Serratosa JM Lafora Disease Offers a Unique Window into Neuronal Glycogen Metabolism. J. Biol. Chem 2018, 293 (19), 7117-7125. 10.1074/jbc.R117.803064. [PubMed: 29483193]

(3). Turnbull J; Tiberia E; Striano P; Genton P; Carpenter S; Ackerley CA; Minassian BA Lafora Disease. Epileptic Disord. 2016, 18 (S2), 38-62. 10.1684/epd.2016.0842. [PubMed: 27702709]

(4). Serratosa JM Idiopathic Epilepsies with a Complex Mode of Inheritance. Epilepsia 1999, 40 (Supple 3), 12-16. [PubMed: 10446745]

(5). Rubio-Villena C; Viana R; Bonet J; Garcia-Gimeno MA; Casado M; Heredia M; Sanz P Astrocytes: New Players in Progressive Myoclonus Epilepsy of Lafora Type. Hum. Mol. Genet 2018, 27 (7), 1290-1300. 10.1093/hmg/ddy044. [PubMed: 29408991]

(6). Augé E; Pelegrí C; Manich G; Cabezón I; Guinovart JJ; Duran J; Vilaplana J Astrocytes and Neurons Produce Distinct Types of Polyglucosan Bodies in Lafora Disease. Glia 2018, 66 (10), 2094-2107. 10.1002/glia.23463. [PubMed: 30152044]

(7). Gentry MS; Romá-Mateo C; Sanz P Laforin, a Protein with Many Faces: Glucan Phosphatase, Adapter Protein, et Alii. FEBS J. 2013, 280 (2), 525-537. 10.1111/j.1742-4658.2012.08549.x. [PubMed: 22364389]

(8). Raththagala M; Brewer MK; Parker MW; Sherwood AR; Wong BK; Hsu S; Bridges TM; Paasch BC; Hellman LM; Husodo S; et al. Structural Mechanism of Laforin Function in Glycogen Dephosphorylation and Lafora Disease. Mol. Cell 2015, 57 (2), 261-272. 10.1016/ j.molcel.2014.11.020. [PubMed: 25544560]

(9). Meekins DA; Raththagala M; Auger KD; Turner BD; Santelia D; Kotting O; Gentry MS; Vander Kooi CW Mechanistic Insights into Glucan Phosphatase Activity against Polyglucan Substrates. J. Biol. Chem 2015, 290 (38), 23361-23370. 10.1074/jbc.M115.658203. [PubMed: 26231210]

(10). Schwarz GA; Yanoff M Lafora's Disease. Distinct Clinico-Pathologic Form of Unverricht's Syndrome. Arch. Neurol. 1965, 12, 172-188. [PubMed: 14237775]

(11). Yokoi S; Austin J; Witmer F Isolation and Characterization of Lafora Bodies in Two Cases of Myoclonus Epilepsy. J. Neuropathol. Exp. Neurol 1967, 26 (1), 125-127. [PubMed: 4164450]

(12). Vilchez D; Ros S; Cifuentes D; Pujadas L; Vallès J; García-Fojeda B; Criado-García O; Fernández-Sánchez E; Medrão-Fernández I; Domínguez J; et al. Mechanism Suppressing Glycogen Synthesis in Neurons and Its Demise in Progressive Myoclonus Epilepsy. Nat. Neurosci 2007, 10 (11), 1407-1413. 10.1038/nn1998. [PubMed: 17952067]

(13). Saez I; Duran J; Sinadinos C; Beltran A; Yanes O; Tevy MF; Martinez-Pons C; Milan M; Guinovart JJ Neurons Have an Active Glycogen Metabolism That Contributes to Tolerance to 
Hypoxia. J Cereb Blood Flow Metab 2014, 34 (6), 945-955. 10.1038/jcbfm.2014.33. [PubMed: 24569689]

(14). Ganesh S; Delgado-Escueta AV; Sakamoto T; Avila MR; Machado-Salas J; Hoshii Y; Akagi T; Gomi H; Suzuki T; Amano K; et al. Targeted Disruption of the Epm2a Gene Causes Formation of Lafora Inclusion Bodies, Neurodegeneration, Ataxia, Myoclonus Epilepsy and Impaired Behavioral Response in Mice. Hum. Mol. Genet 2002, 11 (11), 1251-1262. [PubMed: 12019206]

(15). Turnbull J; DePaoli-Roach AA; Zhao X; Cortez MA; Pencea N; Tiberia E; Piliguian M; Roach PJ; Wang P; Ackerley CA; et al. PTG Depletion Removes Lafora Bodies and Rescues the Fatal Epilepsy of Lafora Disease. PLoS Genet. 2011, 7 (4), 1-10. 10.1371/journal.pgen.1002037.

(16). Turnbull J; Epp JR; Goldsmith D; Zhao X; Pencea N; Wang P; Frankland PW; Ackerley CA; Minassian BA PTG Protein Depletion Rescues Malin-deficient Lafora Disease in Mouse. Ann. Neurol 2014, 75, 442-446. [PubMed: 24419970]

(17). Duran J; Gruart A; Garcia-Rocha M; Delgado-Garcia JM; Guinovart JJ Glycogen Accumulation Underlies Neurodegeneration and Autophagy Impairment in Lafora Disease. Hum Mol Genet 2014, 23 (12), 3147-3156. 10.1093/hmg/ddu024. [PubMed: 24452334]

(18). Brewer MK; Gentry MS The 3rd International Lafora Epilepsy Workshop: Evidence for a Cure. Epilepsy Behav, 2018, 81, 125-127. 10.1016/j.yebeh.2017.12.015. [PubMed: 29475602]

(19). Brewer MK; Grossman TR; McKnight TR; Goldberg YP; Landy H; Gentry MS The 4th International Lafora Epilepsy Workshop: Shifting Paradigms, Paths to Treatment, and Hope for Patients. Epilepsy Behav, 2019, 90, 284-286. 10.1016/j.yebeh.2018.11.014. [PubMed: 30528121]

(20). Chen X; Zaro JL; Shen W-C Fusion Protein Linkers: Property, Design and Functionality. Adv. Drug Deliv, Rev, 2013, 65 (10), 1357-1369. 10.1016/j.addr.2012.09.039. [PubMed: 23026637]

(21). Lee SJ; Chinen J; Kavanaugh A Immunomodulator Therapy: Monoclonal Antibodies, Fusion Proteins, Cytokines, and Immunoglobulins. J. Allergy Clin. Immunol. 2010, 125 (2), S314-S323. 10.1016/j.jaci.2009.08.018. [PubMed: 20036416]

(22). Hansen JE; Weisbart RH; Nishimura RN Antibody Mediated Transduction of Therapeutic Proteins into Living Cells. Sci. World J 2005, 5, 782-788. 10.1100/tsw.2005.98.

(23). Hansen JE; Tse CM; Chan G; Heinze ER; Nishimura RN; Weisbart RH Intranuclear Protein Transduction through a Nucleoside Salvage Pathway. J. Biol. Chem 2007, 282 (29), 2079020793. 10.1074/jbc.C700090200. [PubMed: 17525162]

(24). Zack DJ; Stempniak M; Wong AL; Taylor C; Weisbart RH Mechanisms of Cellular Penetration and Nuclear Localization of an Anti-Double Strand DNA Autoantibody. J. Immunol 1996, 157 (5), 2082-2088. 10.3760/cma.j.issn.1005-1201.2017.07.022. [PubMed: 8757330]

(25). Lawlor MW; Armstrong D; Viola MG; Widrick JJ; Meng H; Grange RW; Childers MK; Hsu CP; O'Callaghan M; Pierson CR; et al. Enzyme Replacement Therapy Rescues Weakness and Improves Muscle Pathology in Mice with X-Linked Myotubular Myopothy. Hum. Mol. Genet. 2013, 22 (8), 1525-1538. [PubMed: 23307925]

(26). Weisbart RH; Gera JF; Chan G; Hansen JE; Li E; Cloninger C; Levine AJ; Nishimura RN A Cell-Penetrating Bispecific Antibody for Therapeutic Regulation of Intracellular Targets. Mol. Cancer Ther 2012, 11 (10), 2169-2173. 10.1158/1535-7163.MCT-12-0476-T. [PubMed: 22863609]

(27). Yi H; Sun T; Armstrong D; Borneman S; Yang C; Austin S; Kishnani PS; Sun B AntibodyMediated Enzyme Replacement Therapy Targeting Both Lysosomal and Cytoplasmic Glycogen in Pompe Disease. J. Mol. Med 2017, 95 (5), 513-521. 10.1007/s00109-017-1505-9. [PubMed: 28154884]

(28). Kishnani P; Lachmann R; Mozaffar T; Walters C; Case L; Appleby M; Libri V; Kak M; Wencel M; Landy H Safety and Efficacy of VAL-1221, a Novel Fusion Protein Targeting Cytoplasmic Glycogen, in Patients with Late-Onset Pompe Disease. Mol. Genet. Metab 2019, 126 (2), S85S86. 10.1016/j.ymgme.2018.12.211.

(29). Hansen JE; Hansen JE; Chan G; Liu Y; Hegan DC; Dalal S; Dray E; Kwon Y; Xu Y; Xu X; et al. Targeting Cancer with a Lupus Autoantibody. Sci. Transl. Med 2012, 4 (157). 10.1126/ scitranslmed.3004385. 
(30). Weisbart RH; Stempniak M; Harris S; Zack DJ; Ferreri K An Autoantibody Is Modified for Use as a Delivery System to Target the Cell Nucleus: Therapeutic Implications. J. Autoimmun, 1998, 11 (5), 539-546. 10.1006/jaut.1998.0212. [PubMed: 9802941]

(31). Weisbart RH; Baldwin R; Huh B; Zack DJ; Nishimura R Novel Protein Transfection of Primary Rat Cortical Neurons Using an Antibody That Penetrates Living Cells. J. Immunol 2000, 164 (11), 6020-6026. 10.4049/jimmunol.164.11.6020. [PubMed: 10820286]

(32). Lu H; Chen C; Klaassen C Tissue Distribution of Concentrative and Equilibrative Nucleoside Transporters in Male and Female Rats and Mice. Drug Metab. Dispos 2004, 32 (12), 1455-1461. 10.1124/dmd.104.001123. [PubMed: 15371301]

(33). Spertini F; Leimgruber A; Morel B; Khazaeli MB; Yamamoto K; Dayer JM; Weisbart RH; Lee ML Idiotypic Vaccination with a Murine Anti-DsDNA Antibody: Phase I Study in Patients with Nonactive Systemic Lupus Erythematosus with Nephritis. J. Rheumatol 1999, 26 (12), 26022608. [PubMed: 10606369]

(34). Nikaido T; Austin J; Stukenbrok H Studies in Myoclonus Epilepsy III. The Effects of Amylolytic Enzymes on the Ultrastructure of Lafora Bodies. J. Histochem. Cytochem 1971, 19 (6), 382-385. 10.1177/19.6382. [PubMed: 4104134]

(35). Brewer MK; Uittenbogaard A; Austin G; McCarthy JJ; Segvich DM; DePaoli-Roach A; Roach PJ; Hodges BL; Zeller J; Pauly JR; et al. Targeting Pathogenic Lafora Bodies in Lafora Disease Using an Antibody-Enzyme Fusion. bioRxiv 2019, 679407 10.1101/679407.

(36). Yokoi S; Austin J; Witmer F; Sakai M Studies in Myoclonus Epilepsy (Lafora Body Form). I. Isolation and Preliminary Characterization of Lafora Bodies in Two Cases. Arch. Neurol 1968, 19 (1), 15-33. [PubMed: 4175641]

(37). Pflüger E Meine Methode Der Quantitativen Analyse Des Glykogenes Und Die Arteigenthümlichkeit Der Substanzen Des Thierleibes. Pflüger, Arch. für die Gesammte Physiol. des Menschen und der Thiere 1909, 129 (6-7), 362-378. 10.1007/BF01677415.

(38). Weisbart R; Noritake D; Wong A; Chan G; Kacena A; Colburn K A Conserved Anti-DNA Antibody Idiotype Associated with Nephritis in Murine and Human Systemic Lupus Erythematosus. J. Immunol 1990, 144 (7), 2653-2658. [PubMed: 2319132]

(39). Sittampalam G; Coussens N; Brimacombe K; Grossman A; Arkin M; Auld D; Austin C; Baell J; Bejcek B; Chung T; et al. Assay Guidance Manual; Sittampalam NP; Brimacombe K; Grossman A; Arkin M; Auld D; Austin C; Baell J; Bejcek B; Chung TDY; Dahlin JL; Devanaryan V; Foley TL; Glicksman M; Hall MD; Hass JV; Inglese J; Iversen PW; Kahl SD; Kales SC; Lal-Nag M; Li Z; McGee J;, Ed. GC; Eli Lilly \& Company and the National Center for Advancing Translational Sciences: Bethesda (MD), 2017.

(40). Asokanathan C; Tierney S; Ball CR; Buckle G; Day A; Tanley S; Bristow A; Markey K; Xing D; Yuen CT An ELISA Method to Estimate the Mono ADP-Ribosyltransferase Activities: E.g in Pertussis Toxin and Vaccines. Anal. Biochem 2018, 540-541 (11 2017), 15-19. 10.1016/ j.ab.2017.10.025.

(41). May JE; Pemberton RM; Hart JP; McLeod J; Wilcock G; Doran O Use of Whole Blood for Analysis of Disease-Associated Biomarkers. Anal. Biochem 2013, 437 (1), 59-61. 10.1016/ j.ab.2013.02.024. [PubMed: 23467100]

(42). Van Heycop Ten HMW Lafora Disease a Form of Progressive Myoclonus Epilepsy In The Epilepsies. Handbook of Clinical Neurology; Vinken PJ, Bruyn GW, Eds.; North-Holland Publishers: Amsterdam, 1975; pp 382-422.

(43). Cohen-pfeffer JL; Gururangan S; Lester T; Lim DA; Shaywitz AJ; Westphal M; Slave I Intracerebroventricular Delivery as a Safe , Long-Term Route of Drug Administration. Pediatr. Neurol 2017, 67, 23-35. [PubMed: 28089765]

(44). Slavc I; Cohen-Pfeffer JL; Gururangan S; Krauser J; Lim DA; Maldaun M; Schwering C; Shaywitz AJ; Westphal M Best Practices for the Use of Intracerebroventricular Drug Delivery Devices. Mol. Genet. Metab 2018, 124 (3), 184-188. 10.1016/J.YMGME.2018.05.003. [PubMed: 29793829]

(45). Cherukuri A; Cahan H; de Hart G; Van Tuyl A; Slasor P; Bray L; Henshaw J; Ajayi T; Jacoby D; O’Neill CA; et al. Immunogenicity to Cerliponase Alfa Intracerebroventricular Enzyme Replacement Therapy for CLN2 Disease: Results from a Phase 1/2 Study. Clin. Immunol 2018, 197, 68-76. 10.1016/j.clim.2018.09.003. [PubMed: 30205177] 
(46). Johnson TB; Cain JT; White KA; Ramirez-Montealegre D; Pearce DA; Weimer JM Therapeutic Landscape for Batten Disease: Current Treatments and Future Prospects. Nat. Rev. Neurol 2019 10.1038/s41582-019-0138-8.

(47). Watts RJ; Zuchero JY; Crouch J; Dennis Mark. Methods for Improving Safety of Blood-Brain Barrier Transport, 1 31, 2019.

(48). Wolak DJ; Pizzo ME; Thorne RG Probing the Extracellular Diffusion of Antibodies in Brain Using in Vivo Integrative Optical Imaging and Ex Vivo Fluorescence Imaging. J. Control. Release 2015, 197, 78-86. 10.1016/J.JCONREL.2014.10.034. [PubMed: 25449807]

(49). Pizzo ME; Wolak DJ; Kumar NN; Brunette E; Brunnquell CL; Hannocks M-J; Abbott NJ; Meyerand ME; Sorokin L; Stanimirovic DB; et al. Intrathecal Antibody Distribution in the Rat Brain: Surface Diffusion, Perivascular Transport and Osmotic Enhancement of Delivery. J. Physiol 2018, 596 (3), 445-475. 10.1113/JP275105. [PubMed: 29023798]

(50). Abbott NJ; Pizzo ME; Preston JE; Janigro D; Thorne RG The Role of Brain Barriers in Fluid Movement in the CNS: Is There a 'Glymphatic' System? Acta Neuropathol. 2018, 135 (3), 387407. 10.1007/s00401-018-1812-4. [PubMed: 29428972]

(51). Kumar NN; Pizzo ME; Nehra G; Wilken-Resman B; Boroumand S; Thorne RG Passive Immunotherapies for Central Nervous System Disorders: Current Delivery Challenges and New Approaches. Bioconjug. Chem. 2018, 29 (12), 3937-3966. 10.1021/acs.bioconjchem.8b00548. 

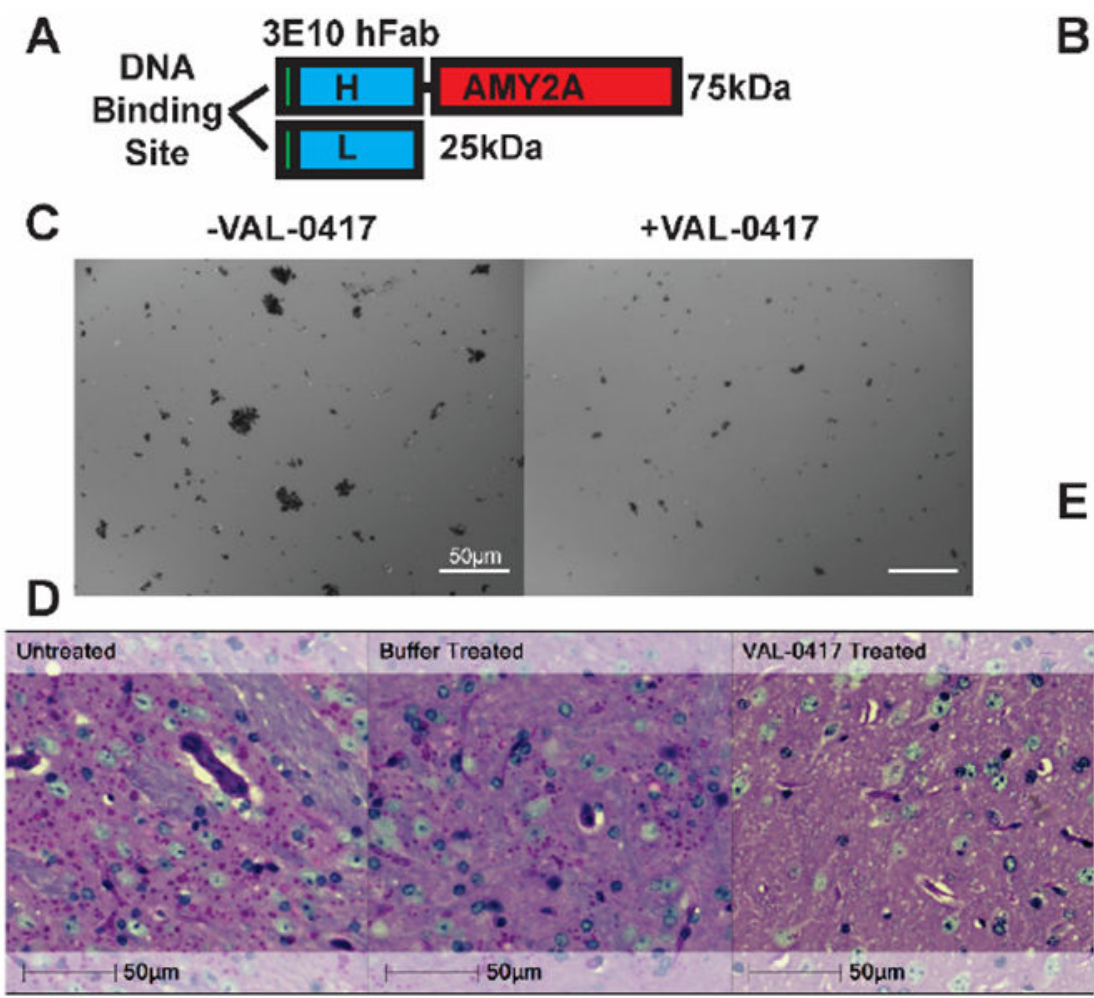

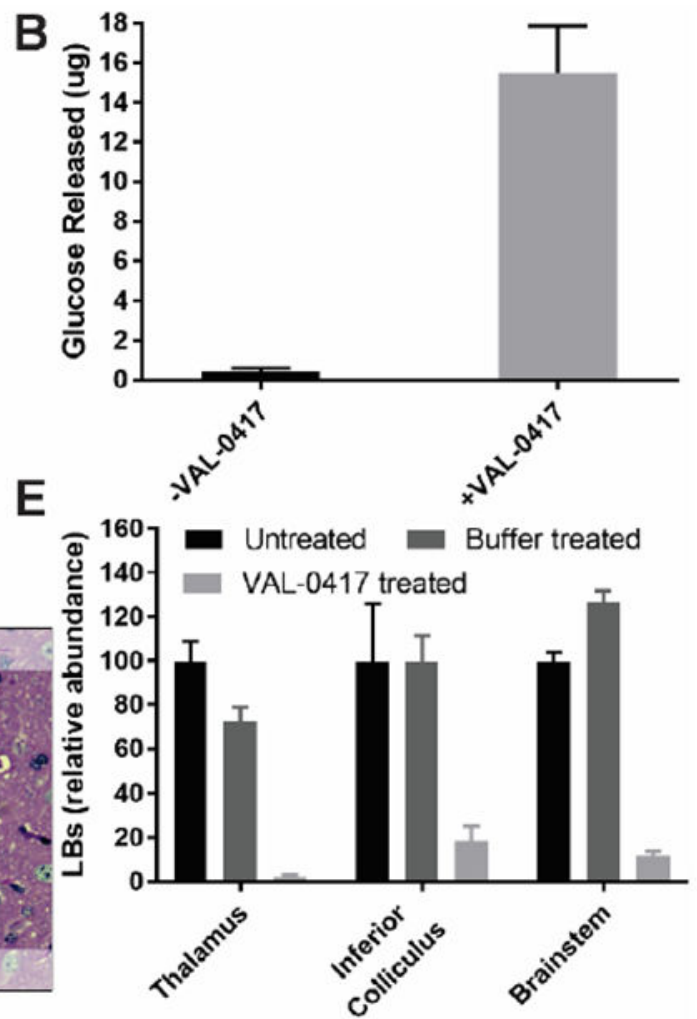

Figure 1:

VAL-0417 illustration and in vitro and ex vivo degradation assays. A. Schematic of VAL-0417 demonstrating the hFab with its DNA binding site that is necessary for cell entry and the amylase protein that degrades LBs. H: heavy chain fragment of auto-antibody 3E10 Fab. L: light chain fragment of auto-antibody 3E10 Fab. AMY2A: human pancreatic aamylase. B. $50 \mu \mathrm{g}$ of brain LBs were treated with $10 \mu \mathrm{g}$ of VAL-0417 in a pH 7.0 buffer and LB degradation was assessed by quantifying the amount of glucose released. C. After incubation in buffer or VAL-0417, LBs were stained with 20x Lugol's solution and visualized by light microscopy. D. Representative images of periodic acid-Schiff (PAS) stained Epm2a-/- mouse brain slices with no treatment, treated with buffer alone, or treated with VAL-0417. PAS+ LBs are pinkish-purple round aggregates visible in the left and middle panels but absent in the right panel. E. Slides from D were analyzed using the HALO digital analysis platform to quantify PAS + LBs in the thalamus, inferior colliculus, and the brain stem. PAS+ LBs are reported as a percent of those identified in untreated samples. 
A 1

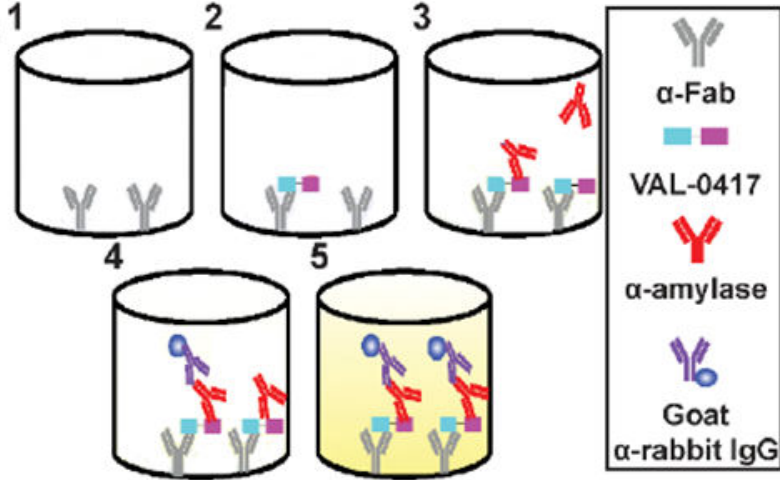

C 0.35

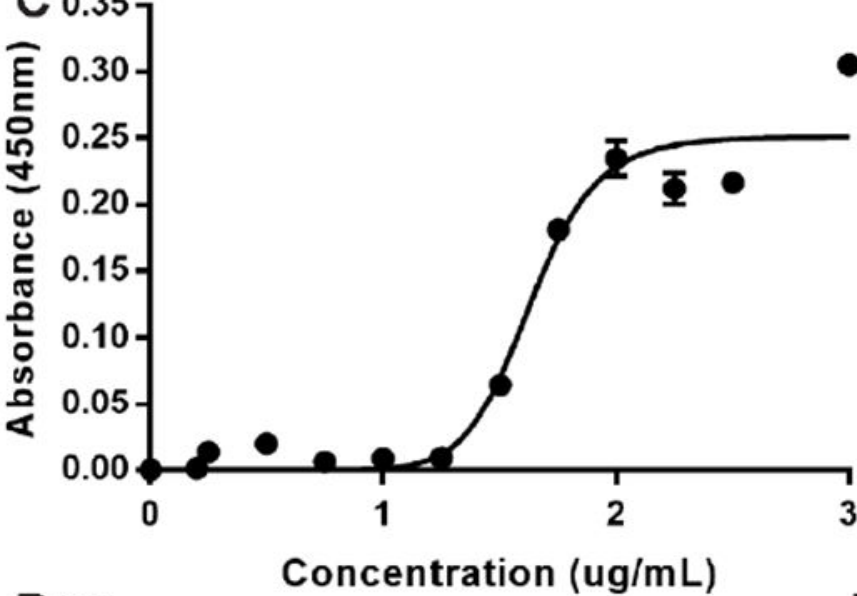

B 150 100 75

50
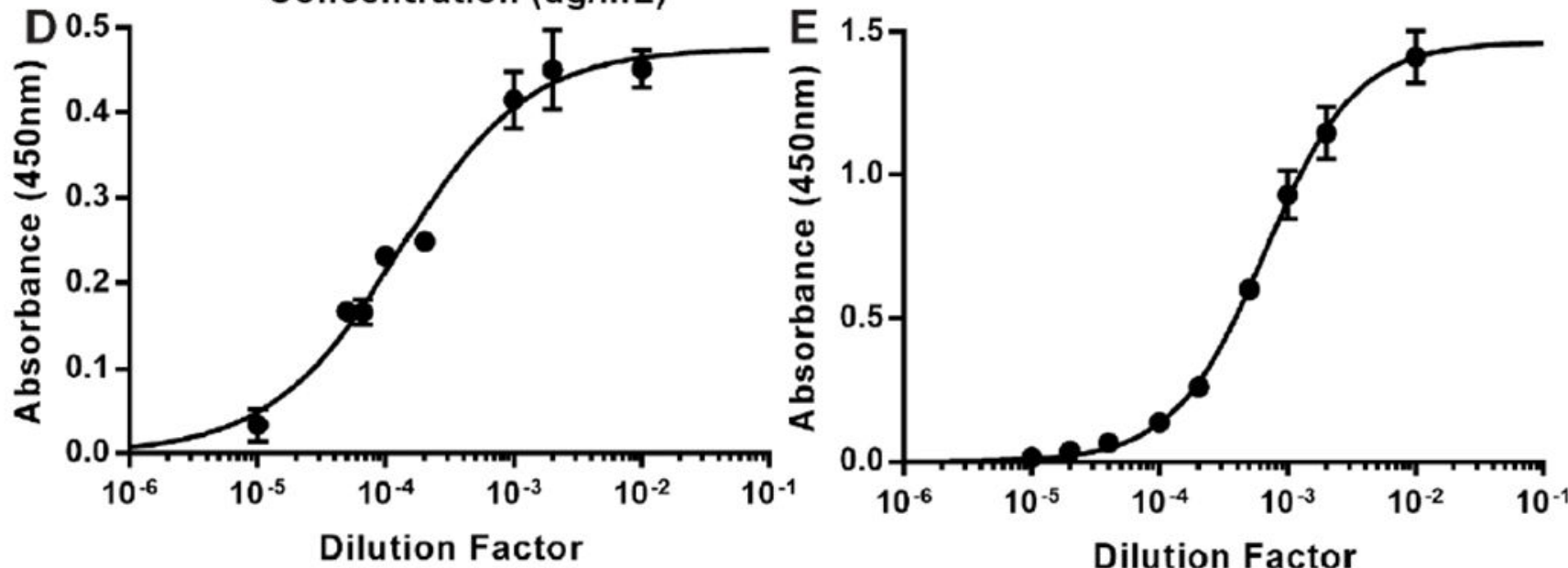

Figure 2:

Optimization of antibodies for the VAL-0417 sandwich ELISA. A: Illustration of the VAL-0417 ELISA. 1: A 96-well plate is coated with the anti-3E10 Fab capture antibody. 2: After blocking, wells are incubated with tissue lysate, some of which contain VAL-0417. 3: VAL-0417 is detected with the Ab21156 rabbit anti-pancreatic amylase antibody. 4: The primary antibody bound is detected using a goat anti-rabbit HRP secondary antibody. 5: The quantity of secondary antibody is detected by development with TMB substrate and $\mathrm{H}_{2} \mathrm{SO}_{4}$ to yield a yellow colorimetric product that is quantified at $450 \mathrm{~nm}$. B: Western blots using the 
rabbit anti-pancreatic amylase (top) and the anti-Fab (bottom). $1 \mu \mathrm{g}$ of VAL-0417, salivary $a$-amylase, and pancreatic a-amylase were separated using a non-reducing SDS-PAGE, a Western transfer was performed, and the blot was probed with rabbit anti-pancreatic amylase (ab21156) or anti-3E10 Fab (ab10A4). Blots are representative from $n=4$. C: Determination of binding efficiency of the anti-Fab to a high binding plate. The capture antibody was plated onto a high binding 96-well plate and the amount of antibody adhered after overnight incubation was detected using a goat anti-mouse HRP antibody. The experiment uses $n=3$ replicates. D: Dilution curve of anti-pancreatic amylase antibody in the ELISA with constant concentrations of anti-Fab ( $2 \mathrm{ng} / \mu \mathrm{L})$, VAL-0417 (500 pg/ $\mu \mathrm{L})$, and goat antirabbit HRP secondary antibody (1/5000). The experiment uses $n=3$ replicates. E. Dilution curve of goat anti-rabbit HRP in the ELISA with constant concentrations of anti-Fab (2ng/ $\mathrm{LL}), \mathrm{VAL}-0417$ $(500 \mathrm{pg} / \mu \mathrm{L})$, and anti-pancreatic amylase antibody $(1 / 1000)$. The experiment uses $n=3$ replicates. 

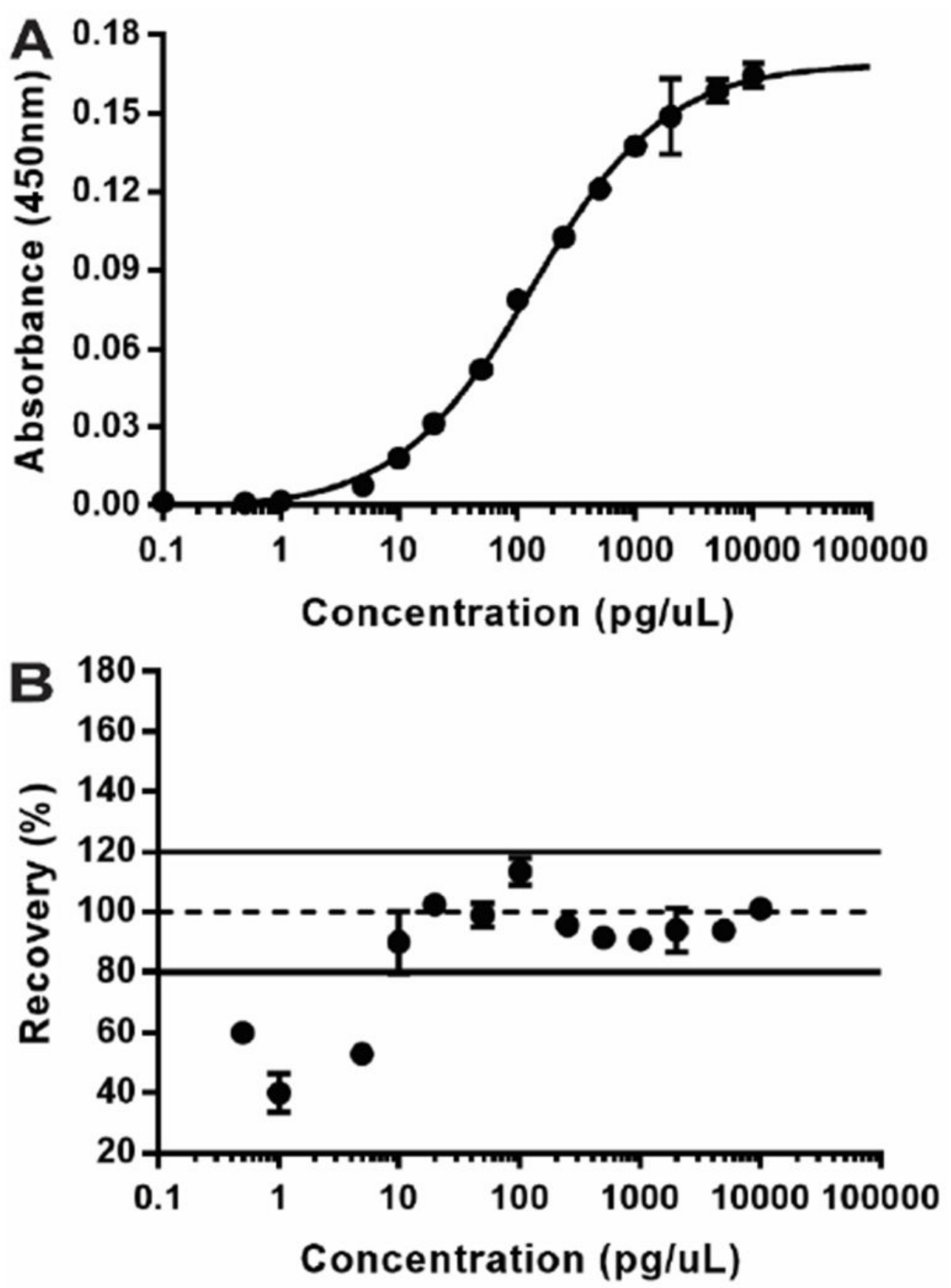

Figure 3:

The working range of VAL-0417 in PBS at optimized antibody concentrations. A. An absorbance versus concentration curve was generated for multiple concentrations to determine the working range of the VAL-0417 ELISA. The minimum detection is $1 \mathrm{pg} / \mu \mathrm{L}$ and signal saturation is reached above $5000 \mathrm{pg} / \mu \mathrm{L}$. The curve is fit with a four-parameter logistic (FPL) best fit curve. The experiment uses $n=3$ replicates. B. Using the FPL model, percent recovery was determined by comparing concentration loaded versus the concentration as determined by the curve. 

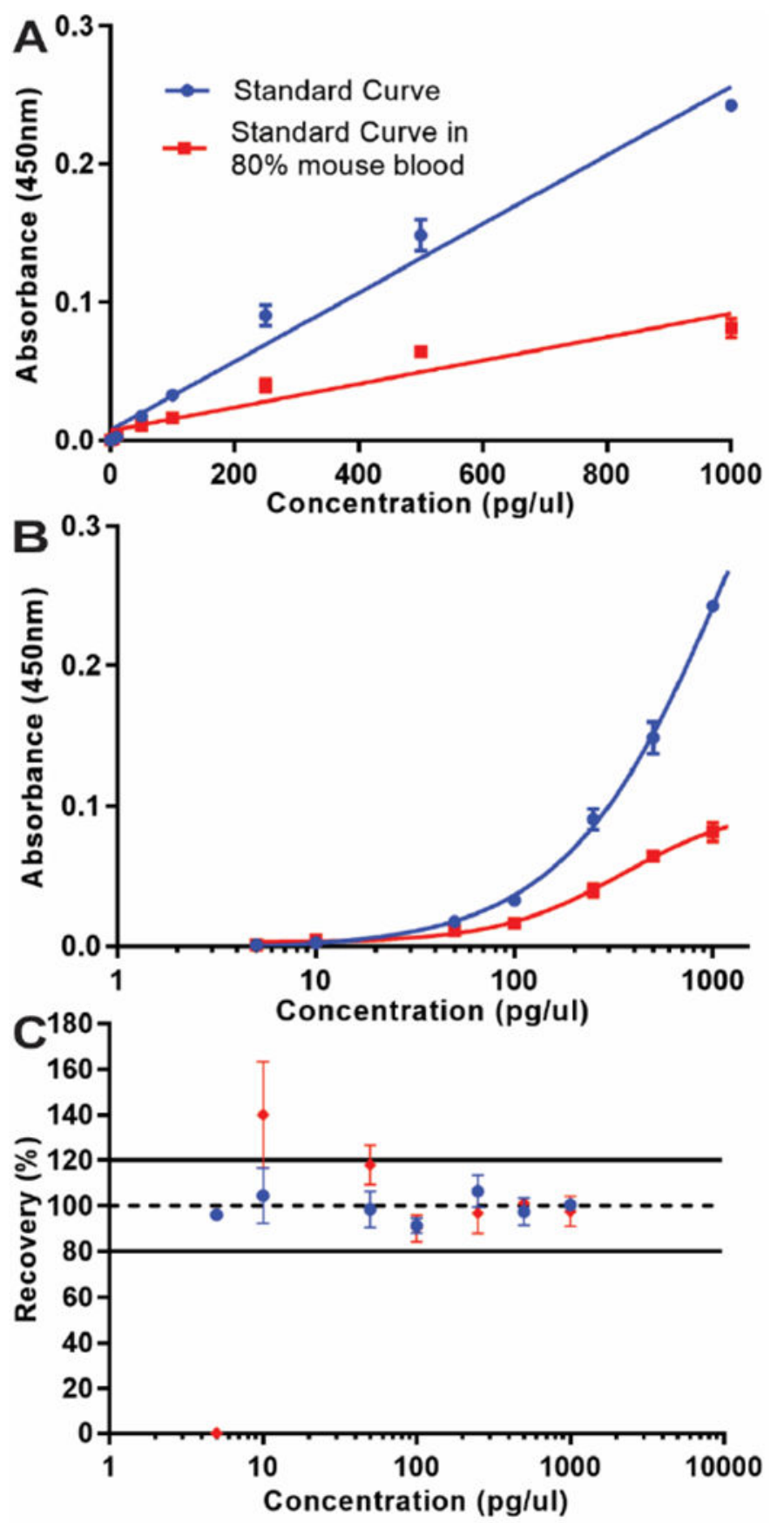

Figure 4:

The effect of whole mouse blood on the VAL-0417 ELISA. A. A standard curve derived from varying concentrations of VAL-0417 in either PBS (Blue) or 80\% whole mouse blood (Red) fit with a linear best fit curve. The experiment uses $n=3$ replicates. B. The same standard curves as in A fit with an FPL best fit curve. C. Percent recovery of both curves against the FPL model best fit curve. 


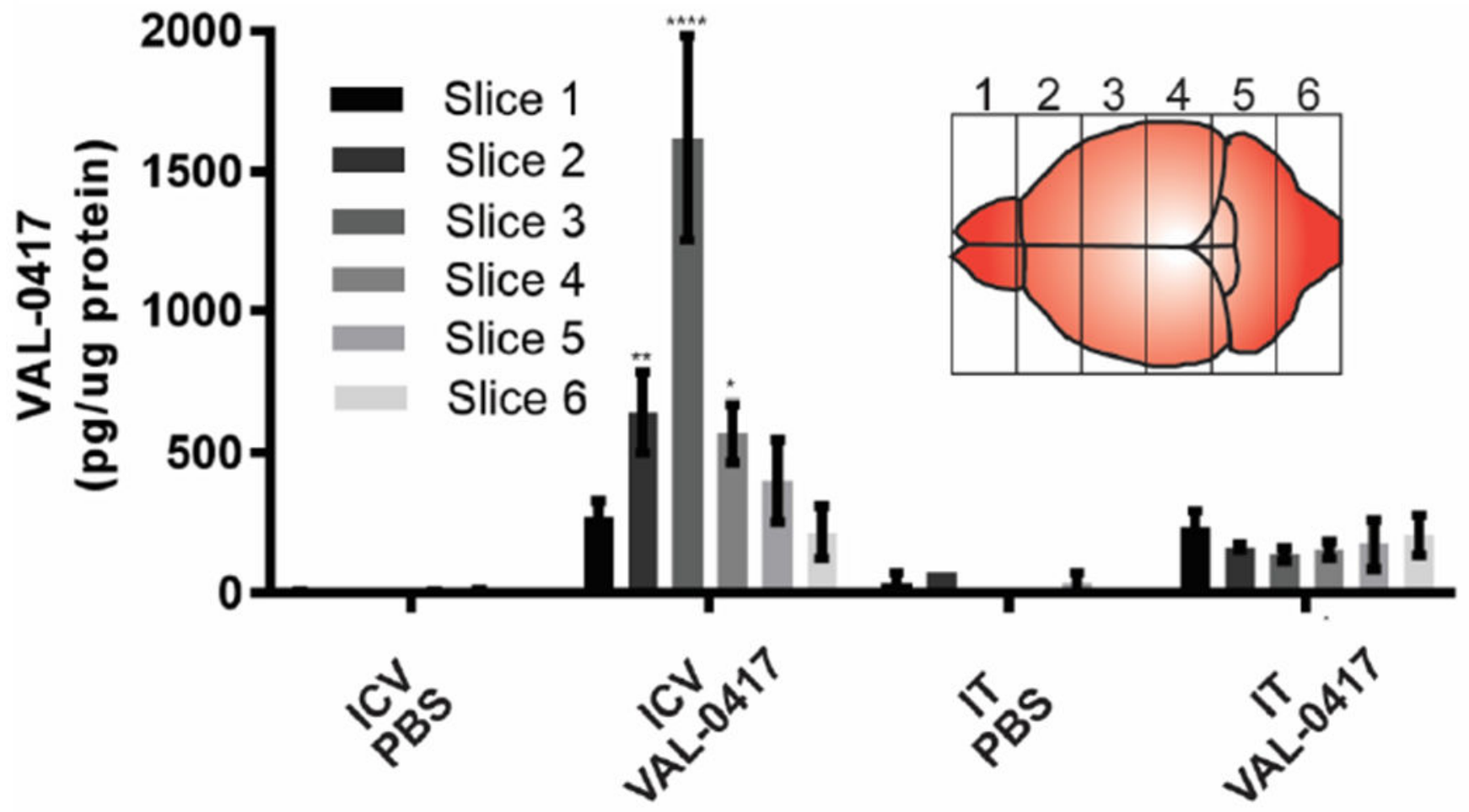

Figure 5:

In vivo quantification of VAL-0417 in brain tissue after VAL-0417 treatment administered via intracerebroventricular (ICV) or intrathecal (IT) administration in WT mice. Distribution by slice of the concentration per $\mu \mathrm{g}$ of protein of VAL-0417 in the brain of WT mice after ICV or IT administration of PBS or VAL-0417 for 14 days. Mice were sacrificed one day following the completion of treatment (day 15) and brains were harvested and sectioned laterally into six slices numbered from rostral to caudal (inset). These data include three mice in each group except the IT PBS group, which had two mice. Significance is measured by two-way ANOVA and is measured against the PBS measurement from the same slice and treatment duration. * is $\mathrm{p} \unlhd 0.05, * *$ is $\mathrm{p} \unlhd .01$, and $* * * *$ is $\mathrm{p} \unlhd 0.0001$. 

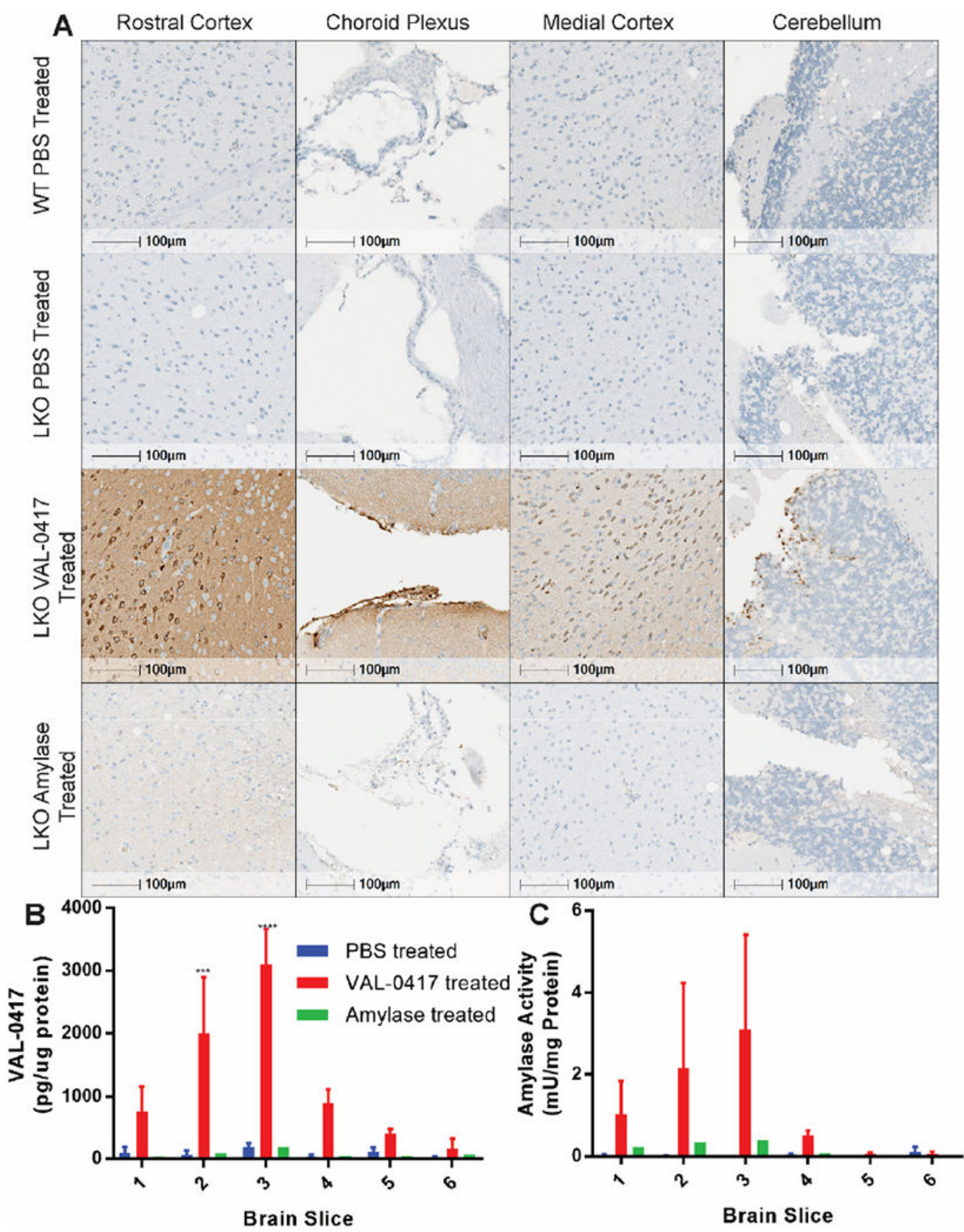

Figure 6:

VAL-0417 delivery in a LD mouse model. A. IHC analysis using an a-amylase antibody. Sections corresponding to multiple brain regions were processed and analyzed for amylase localization from WT mice treated with PBS as well as laforin KO (LKO) mice treated with PBS, VAL-0417, or free a-amylase. These images are representative from two mice in each group. B. The concentration of VAL-0417 in brain tissue after treatment of laforin KO mice by PBS, VAL-0417, or recombinant amylase as determined by the ELISA. Results are presented for each brain slice. C. Amylase activity in brain tissue after treatment of laforin 
KO mice by PBS, VAL-0417, or recombinant amylase. This activity assay provides a confirmation of the ELISA results. Results are presented for each brain slice. These data include three mice each in the PBS and VAL-0417 groups and five mice in the Amylase group. Significance is measured by two-way ANOVA and is measured against the PBS measurement from the same slice. $* * *$ is $\mathrm{p} \unlhd(001, * * * *$ is $\mathrm{p} \unlhd 0.0001$. 